\title{
Diagnóstico radiográfico e tomográfico de osteocondrose dissecante em tarso de cão
}

\author{
Radiographic and Tomographic Diagnosis of Tarsal Osteochondrosis Dissecans in Dog

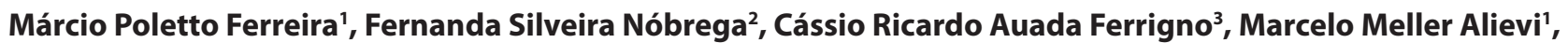 \\ Luciana Zang ${ }^{4}$, Mariana de Mattos Brose ${ }^{4}$, Mariana de Jesus de Souza ${ }^{4}$ \& Rafaela Scheer Bing ${ }^{4}$
}

\begin{abstract}
Background: The tarsal joint is the third most commonly joint affected by osteochondrosis in dogs. This joint is affected in 4 to $9 \%$ of the cases, and in $50 \%$ of these cases the disease is bilateral. Radiological signs include defect in the subchondral bone, sclerotic margin, and fragment of mineralized cartilage. Dorsoplantar in flexion and oblique radiologic images are necessary to show tarsal osteochondrosis. Computed tomography avoid overlap of bone structures and allows better visualization of subchondral lesions, showing the exact location, size and number of fragments. The objective of this study is to report a case of bilateral osteochondrosis dissecans in tarsal joint in Rottweiler.

Case: A female Rottweiler was examined and presented a history of pain and difficulty in raising the pelvic limbs. There was pain in the right tibiotarsal joint (RTJ) with evident joint cracking and, after three months, the left pelvic limb started to show the same signs. Radiographic examination of the RTJ showed a small osteochondral fragment in the distal medial border of the medial malleolus of the tibia, subchondral sclerosis with radiolucent area in the medial malleolus of the tibia, and a small lateral periarticular osteophyte in the intertarsal joint. The left tibiotarsal joint (LTJ) showed mild articular incongruity and minor increase in radiological interlinear space, diffuse sclerosis in the medial malleolus and in the subchondral surface of the trochlear crest. This exam also showed a small radiolucent area in the medial malleolus of the tibia, a small fragment of radiopaque adjacent bone, and minor subchondral bone irregularity in the medial margin of the medial trochlea. The radiographic examination of the RTJ was inconclusive. Computed tomography was requested and suggested the diagnosis of medial osteochondritis dissecans in the distal talus. The most important tomographic signs were irregularity of the subchondral bone in the caudal region of the distal talus associated to isolated bone fragment, irregularity of the distal and caudal portion of the medial malleolus of the tibia associated with the presence of free adjacent osteophyte. Discussion: Only 4 to $9 \%$ of the dogs that develop osteochondrosis present this lesion in the tarsus. The Rottweiler is the breed most affected by this disease, such as the subject of this case report, and is also the breed most frequently affected in the lateral region of the tarsus. It is a fast-growing large breed, a common characteristic in animals affected by osteochondrosis. Bilateral cases are most common in young dogs, such as the patient of this case report. Clinical manifestation of pain and lameness is frequent in this type of disease, and lameness can occur in only one of the affected limbs. The dog of this study presented lesion in the medial region of the trochlea of the talus, one of the regions that present high incidence of osteochondrosis. Radiographic examination was efficient to suggest lesions in the medial region of the talus. However, only $10 \%$ of the dogs with osteochondrosis of the tarsus are diagnosed by this technique. The definitive diagnosis was not provided by radiographic examinations in this patient. Computed tomography has several advantages over conventional radiography, such as eliminating overlap of structures, decreasing image complexity, and increasing the ability to identify specific structures. This examination allowed us to reach the diagnosis of osteochondrosis dissecans in the right talus of this dog. A scan was not performed on the contralateral limb (LTJ), because the lesions observed on radiographic examination were similar to the lesions observed in the right pelvic limb.
\end{abstract}

Keywords: canine, radiology, computed tomography, orthopedics.

Descritores: cão, radiologia, tomografia computadorizada, ortopedia.

${ }^{1}$ Departamento de Medicina Animal, Faculdade de Veterinária (FaVet), Universidade Federal do Rio Grande do Sul (UFRGS), Porto Alegre, RS, Brazil. ${ }^{2}$ M.V. autônoma, Porto Alegre. ${ }^{3}$ Departamento de Cirurgia, Faculdade de Medicina Veterinária e Zootecnia da Universidade de São Paulo (FMVZ-USP), São Paulo, SP, Brazil. ${ }^{4}$ Programa de Pós-graduação em Ciências Veterinárias (PPG-CV), FaVet, UFRGS. CORRESPONDENCE: M.P. Ferreira [marcio.ferreira@ ufrgs.br - Tel.: +55 (51) 33087856]. Faculdade de Veterinária - UFRGS. Av. Bento Gonçalves n.9090. Bairro Agronomia. CEP 91540-000 Porto Alegre, RS, Brazil. 


\section{INTRODUÇÃO}

A osteocondrose (OC) é alteração comum no crescimento em animais [2]. Pode ser latente, manifesta ou dissecante (OCD), sendo a OCD a manifestação clínica da osteocondrose [15]. Formam-se fissuras na área de cartilagem necrosada que podem gerar "flap" ou fragmentos soltos [20], provocando processo inflamatório crônico $[13,16]$.

A articulação do tarso é a terceira articulação mais comumente acometida por osteocondrose no cão, com 4 a 9\% dos pacientes [14], podendo ser dividida em alteração na porção medial (mais comum) ou na porção lateral [7]. Aproximadamente 50\% dos pacientes apresentam a doença de forma bilateral [19]. Rottweiler tem maior ocorrência desta lesão na porção lateral da articulação [3] e é a raça mais frequentemente acometida, sendo esta doença mais comum em cães de grande porte [8].

O diagnóstico desta afecção pode ser realizado através de exame radiográfico, exame tomográfico [5] e ressonância magnética [18]. Podem ser observados como sinais radiológicos defeito no osso subcondral com margem esclerótica, fragmento pediculado solto e calcificado e fragmentos livres mineralizados na cartilagem [8]. A incidência dorsoplantar flexionada do tarso é necessária para demonstrar osteocondrose no tarso, sendo recomendadas também as incidências oblíquas com rotação medial e lateral [8]. A tomografia computadorizada evita a sobreposição de estruturas ósseas e permite melhor visualização das lesões ósseas subcondrais, identificando a localização exata, tamanho e número de fragmentos [8,9]. O objetivo deste trabalho é relatar um caso de osteocondrite dissecante em articulação bilateral de tarso em um canino da raça Rottweiler.

\section{CASO}

Um canino, fêmea, castrada, da raça Rottweiler, com um ano de idade e $32,3 \mathrm{~kg}$ de massa corporal foi atendido no Laboratório de Ortopedia e Traumatologia Comparada do Departamento de Cirurgia da Faculdade de Medicina Veterinária e Zootecnia da Universidade de São Paulo (FMVZ/USP), com histórico de dor e dificuldade para levantar com os membros pélvicos.

Ao exame físico foi observado dor em articulação tibiotársica direita (ATD), com evidente crepitação. Não foram observadas outras alterações ortopédicas neste ou nos outros membros. Foi solicitado exame

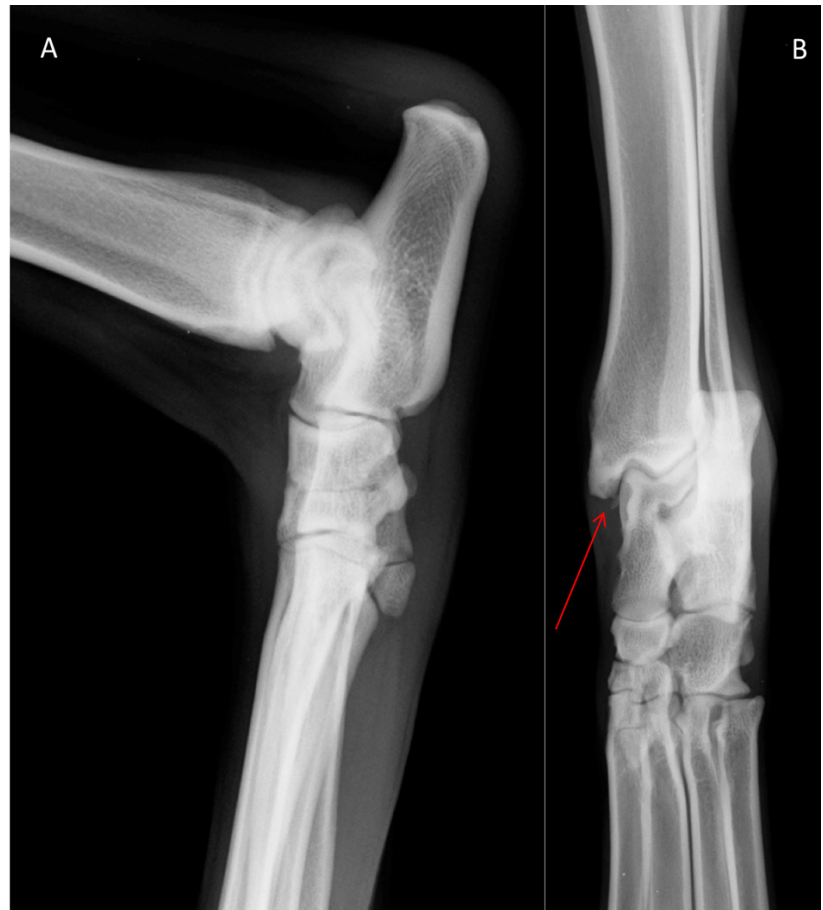

Figura 1. Imagem radiográfica do tarso direito de canino nas projeções mediolateral com a articulação flexionada (A) e dorsoplantar com a articulação estendida (B), evidenciando pequeno fragmento osteocondral junto a borda mediodistal do maléolo medial da tíbia (seta).

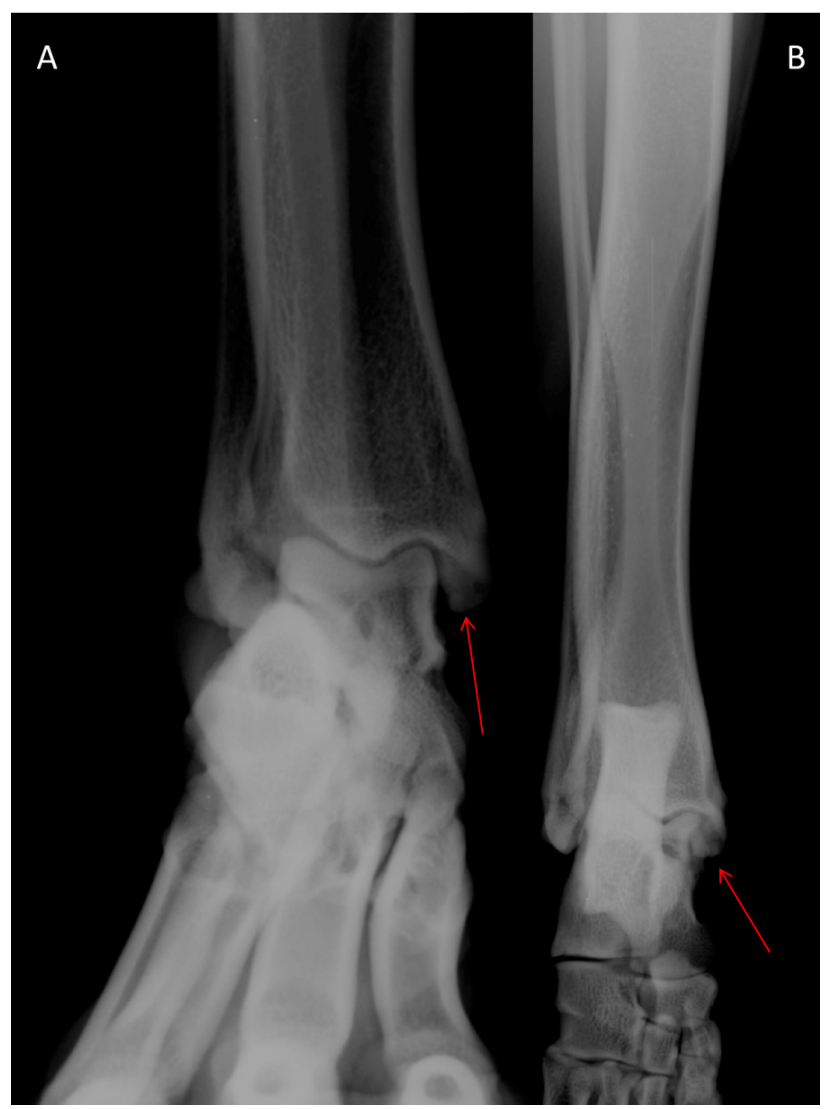

Figura 2. Imagem radiográfica do tarso direito de canino nas projeções dorsoplantar com a articulação flexionada (A) e dorsoplantar com a articulação estendida (B), evidenciando pequena irregularidade óssea subcondral na margem medial da tróclea medial e pequeno fragmento ósseo arredondado entre a tróclea e o maléolo medial (setas). 


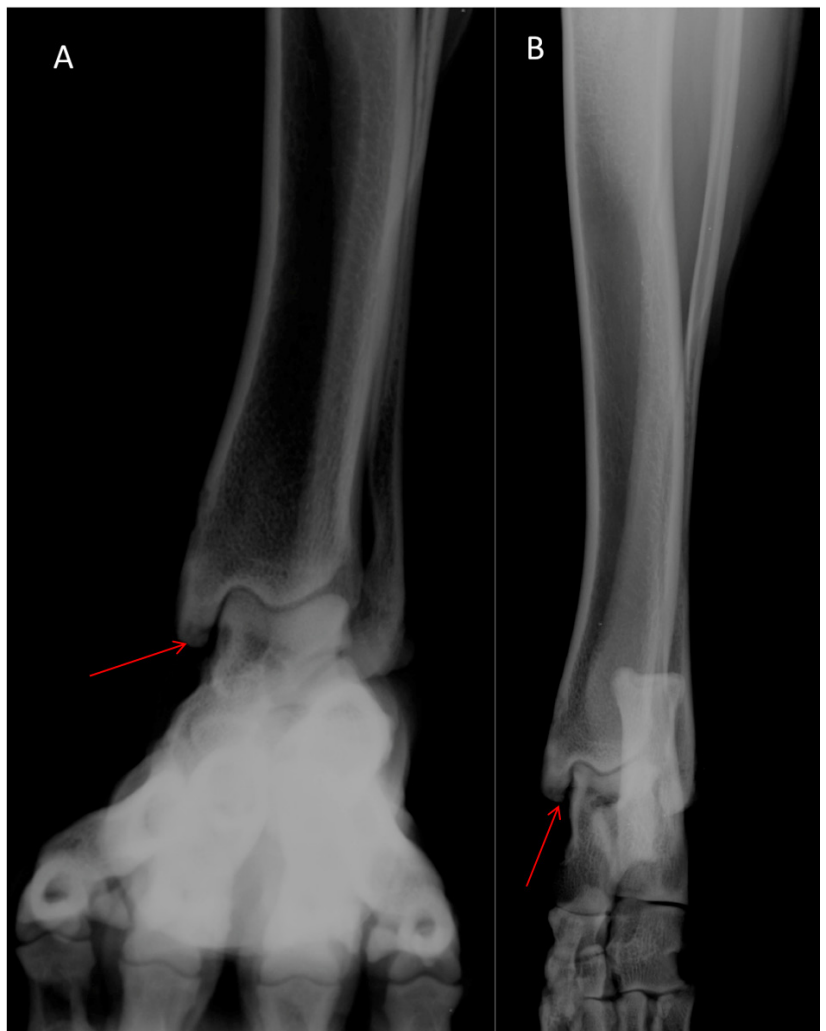

Figura 3. Imagem radiográfica do tarso esquerdo de canino nas projeções dorsoplantar com a articulação flexionada (A) e dorsoplantar com a articulação estendida (B) evidenciando pequena irregularidade óssea subcondral na margem medial da tróclea medial (seta).

radiográfico da $\mathrm{ATD}$, onde foi constatado pequeno fragmento osteocondral junto a borda mediodistal do maléolo medial da tíbia; esclerose subcondral com área de radiotransparência em maléolo medial da tíbia e pequeno osteofito periarticular lateral em articulação intertársica proximal (Figura 1). Este exame foi realizado sem sedação e nas projeções dorsoplantar (articulação em extensão) e mediolateral (articulação em flexão de $+/-90^{\circ}$ ).

Após três meses do primeiro atendimento, iniciou com crepitação e dor na articulação tibiotársica esquerda (ATE), manifestação clínica semelhante à observada em ATD. Foi solicitado exame radiográfico de ambas as articulações tibiotársicas, no qual foi observado articulação tibiotársica direita com discreta incongruência articular e com pequeno aumento da interlinha radiográfica, esclerose difusa em maléolo medial e em superfície subcondral das cristas trocleares. Pequena irregularidade óssea subcondral na margem medial da tróclea medial. Pequeno fragmento ósseo arredondado (mede cerca de $0,3 \mathrm{~cm}$ de diâmetro) entre a tróclea e o maléolo medial. Pequeno osteófito periarticular intertársico proximal na face lateral (Figura 2). Na ATE havia discreta incongruência articular e pequeno aumento da interlinha radiográfica, esclerose difusa em maléolo medial e em superfície subcondral das cristas trocleares. Pequena área de radiotransparência em maléolo medial da tíbia e pequeno fragmento de radiopacidade óssea adjacente (mede $<0,1 \mathrm{~cm}$ de diâmetro). Pequena irregularidade óssea subcondral na margem medial da tróclea medial (Figura 3). Não foram sugeridos diagnósticos para estas alterações. Este exame foi realizado sem sedação e nas projeções dorsoplantar com a articulação em extensão, dorsoplantar com a articulação em flexão e mediolateral (articulação em flexão de $+/-90^{\circ}$ ).

Foi solicitado, após o atendimento inicial, tomografia computadorizada da articulação tibiotársica e tarso direito, na qual foi observada irregularidade do osso subcondral na região caudomedial do talo distal, associado ao desprendimento de "flap" ósseo, que media $0,4 \mathrm{~cm}$ x 0,2 cm. Irregularidade da porção distal e caudal do maléolo medial da tíbia direita, associada à presença de osteófito livre adjacente, que mede 0,24 $\mathrm{cm}$ x $0,13 \mathrm{~cm}$ x 0,3 cm. Diminuto osteófito livre caudal a região proximal do quinto metatársico. Presença de diminuta proliferação óssea cortical na face medial da extremidade proximal do segundo metatársico, com presença de diminutos osteófitos entre o primeiro társico e o segundo metatársico. Diminuto osteófito caudal ao terceiro társico. Diminuta linha de fissura (Figura 4) na região craniolateral da epífise distal do calcâneo.

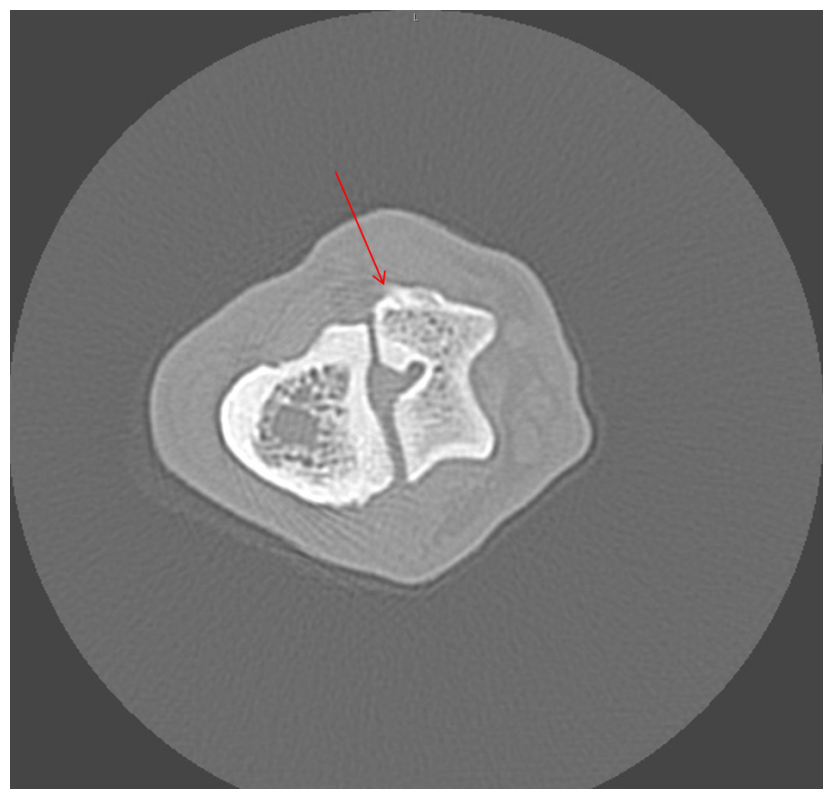

Figura 4. Imagem tomográfica do tarso direito de canino realizado com a articulação estendida e pelo método multislice e colimação 4 X0,5 mm, evidenciando lesão de osteocondrite dissecante em região medial, com diminuta linha de fissura (seta) na região craniolateral da epífise distal do calcâneo. 
A técnica utilizada neste paciente foi método helicoidal multislice e colimação de $4 \mathrm{X} 0,5 \mathrm{~mm}$, sendo sugerido o diagnóstico de osteocondrite dissecante medial do talo.

\section{DISCUSSÃO}

Apenas 4 a $9 \%$ dos cães que desenvolvem osteocondrose apresentam esta lesão no tarso [14], o que torna este caso incomum, mesmo que a região acometida seja a mais frequente para osteocondrose [7].

Dentre as raças mais acometidas por esta afecção está o Rottweiler, como o paciente deste relato [1], sendo esta raça, mais frequentemente acometida na região lateral do tarso [8]. Esta é considerada raça de grande porte e crescimento rápido, características comuns em animais com esta afecção [12,20]. Os machos são mais acometidos que as fêmeas [12], porém, neste, trabalho foi diagnosticado em uma fêmea.

É mais comum a ocorrência bilateral [11] e em cães jovens [10] como observado neste relato. A manifestação clínica de dor e claudicação é comum neste tipo de doença, sendo possível observar claudicação em apenas um dos membros acometidos, fato observado neste cão. O cão deste trabalho apresentou lesão na região medial da tróclea do talo, sendo esta uma das regiões consideradas com alta incidência de osteocondrose [20].

O exame radiográfico foi eficiente em sugerir alterações na região medial do talo, porém, apenas $10 \%$ dos cães com osteocondrose do tarso são diag- nosticados com esta técnica [8,9], não sendo fornecido diagnóstico definitivo desta doença pelos exames radiográficos realizados neste paciente. Esta é informação fundamental para a tomada de decisão entre intervir cirurgicamente ou não.

A tomografia computadorizada apresenta diversas vantagens em comparação com a radiografia convencional, como eliminar a sobreposição de estruturas, diminuindo a complexidade da imagem e aumentando a capacidade de identificar estruturas específicas [6] e este exame permitiu definir o diagnóstico de ostecondrose dissecante do talo direito neste paciente. Este mesmo exame não foi realizado no membro contralateral (membro pélvico esquerdo), pois as alterações observadas ao exame radiográfico foram semelhantes às observadas no membro pélvico direito e este exame ainda apresenta custo elevado.

Concluiu-se que os exames radiográficos nas projeções mediolateral e dorsoplantar foram eficientes em sugerir alteração em compartimento medial do talo nas articulações tibiotársicas direita e esquerda do paciente deste relato e que a tomografia computadorizada foi eficaz em definir o diagnóstico de ostecondrose dissecante medial do talo na articulação do tarso do membro pélvico direito.

Declaration of interest. The authors report no conflicts of interest. The authors alone are responsible for the content and writing of the paper.

\section{REFERENCES}

1 Alvarenga J., Garcia J.A., Iwasaki M. \& Laus J.L. 1993. Osteocondrite dissecante da cabeça do úmero. A Hora Veterinária. 73(2): 68-74.

2 Bohndorf K. 1998. Osteochondritis (osteochondrosis) dissecans: a review and new MRI classification. European Radiology. 8(1): 103-112.

3 Carlisle C.H., Robins G.M. \& Reynolds K.M. 1990. Radiographic signs of osteochondritis dissecans of the lateral ridge of the trochlear talus in the dog. Journal of Small Animal Practice. 31(6): 280-286.

4 Carlson C.S., Hilley H.D. \& Meuten D.J. 1989. Degeneration of cartilage canal vessels associated with lesions of osteochondrosis in swine. Veterinary Pathology. 26(1): 47-54.

5 Dingemanse W.B., Van Bree H.J.J., Duchateau L. \& Gielen I.M.V.L. 2013. Comparison of clinical and computed tomographic features between medial and lateral trochlear ridge talar osteochondrosis in dogs. Veterinary Surgery. 42(3): 340-345.

6 Drost W.T., Love N.E. \& Berry C.R. 1996. Comparison of radiography, myelography and computed tomography for thee valuation of canine vertebral and spinal cord tumors in sixteen dogs. Veterinary Radiology and Ultrasound. 37(1): 28-33.

7 Fitch R.B. \& Beale B.S. 1998. Osteochondrosis of the canine tibiotarsal joint. Veterinary Clinics of North America: Small Animal Practice. 28(1) 95-113.

8 Gielen I., Van Bree H., Van Ryssen B., De Clercq T. \& Rooster H. 2002. Radiographic, computed tomographic and arthroscopic findings in 23 dogs with osteochondrosis of the tarsocrural joint. Veterinary Record. 150(14): 442-447. 
9 Gielen I., Van Ryssen B. \& Van Bree H. 2005. Tomography compared with radiography in the diagnosis of lateral trochlear ridge osteochondritis dissecans in dogs. Veterinary and Comparative Orthopaedics and Traumatology. 18(1): 77-81.

10 Harari J. 1998. Osteochondrosis. Veterinary Clinics of North America: Small Animal Practice. 28(1): 1-195.

11 Jørgensen B. \& Andersen S. 2000. Genetic parameters for osteochondrosis in Danish Landrace and Yorkshire boars and correlations with leg weakness and production traits. Animal Science. 71(3): 427-434.

12 Leighton R.L. 1998. Historical perspectives of osteochondrosis. Veterinary Clinics of North America: Small Animal Practice. 28(1): 1-16.

13 Martinez S.A. 1997. Congenital conditions that lead to osteoarthritis in the dog. Veterinary Clinics of North America: Small Animal Practice. 27(4): 735-757.

14 Necas A., Dvorak M. \& Zatloukal J. 1999. Incidence of osteochondrosis in dogs and it's late diagnosis. Acta Veterinaria. 68(1): 131-139.

15 Ohlerth S., Senn S., Geissbühler U., Kircher P. \& Flückiger M. 2016. Prevalence of humeral head osteochondrosis in the Greater Swiss Mountain dog and the Border Collie in Switzerland. Schweizer Archiv für Tierheilkunde. 158(11): 749-754.

16 Rudd R.G., Whitehair J.G. \& Margolis J.H. 1990. Results of management of osteocondritis dissecans of the umeral head in dogs: 44 cases (1982 to 1987). Journal of American Animal Hospital Association. 26(2): 173-178.

17 Smith M., Vasseur P. \& Morgan J. 1985. Clinical evaluation of dogs after surgical and non surgical management of osteochondritis dissecans of the talus. Journal of the American Veterinary Medical Association. 187(1): 31-35.

18 Van Bree H., Gielen I. \& Van Ryssen B. 2002. Comparative joint imaging in small animal. European Journal of Companion Animal Practice. 12(1): 25-36.

19 Weinstein M.J., Mongil C.M. \& Rhodes W.H. 1995. Orthopedic conditions of the Rottweiler-Part II. Compendium on Continuing Education for the Practicing Veterinarian. 17(7): 925-939.

20 Ytrehus B., Carlson C.S. \& Ekman. S. 2007. Etiology and pathogenesis of osteochondrosis. Veterinary Pathology. 44(4): 429-448. 